This is a peer-reviewed, accepted author manuscript of the following research article: Moreno, R., Trakas, D. N., Jamieson, M., Panteli, M., Mancarella, P., Strbac, G., Marnay, C., \& Hatziargyriou, N. (2022). Microgrids against wildfires: distributed energy resources enhance system resilience. IEEE Power and Energy Magazine, $20(1), 78-89$. https://doi.org/10.1109/MPE.2021.3122772

\title{
Microgrids against Wildfires: Distributed Energy Resources Enhance System Resilience
}

Rodrigo Moreno, Dimitris N. Trakas, Magnus Jamieson, Mathaios Panteli, Pierluigi Mancarella, Goran Strbac, Chris Marnay, and Nikos Hatziargyriou

In recent years, countries around the world have been severely affected by catastrophic wildfires with significant environmental, economic, and human losses. Critical infrastructures, including power systems, have been severely damaged, compromising the quality of life and the continuous and reliable provision of essential services, including the electricity supply.

When such disasters strike, the impacts usually go beyond what the system has been designed to withstand, potentially leading to prolonged power outages for large numbers of customers and critical loads in the system. These impacts are expected to only get worse as a result of climate change-driven high temperatures in wildfire seasons as recently highlighted in California, Australia, Chile, Brazil, Portugal, Italy, and Greece.

Power system planners have traditionally attempted to boost the resilience of critical electrical power infrastructure against such high-impact low-probability events by making the network redundant or stronger to withstand their severe shocks. Nevertheless, recent wildfires reiterate that alternative solutions need to be explored and deployed for the holistic provision of robustness, preparedness, and recovery. Distributed energy resources (DERs) and microgrids arise as attractive decentralized options for providing a means for riding through and recovering from the catastrophic impacts of wildfires. They represent localized energy solutions that are potentially less exposed to the wildfire effects compared to network assets.

This article aims to tackle a set of key relevant questions:

- What is the role of DERs and microgrids to protect the system against wildfires?

- How can microgrid operational capabilities improve traditional reliability-driven approaches for making the network more resilient?

- How can planners assess investments in DER portfolios to achieve an effective trade-off between DERs and network planning?

- What are the regulatory and policy barriers in adapting such hybrid resilience enhancement portfolios against wildfires? 
This article provides an overview of real-world evidence to understand the potential contribution of DERs and microgrids against wildfires. It presents a holistic framework for assessing and quantifying the role of DERs in operational planning and investment decision-making to enable additional robustness and flexibility in a system exposed to wildfires.

\section{Real-world examples}

Looking at real-world examples can help explore the potential benefits of DERS and microgrids to improve power system resilience under wildfire scenarios. Using challenging situations where these resources have mitigated or resolved operational issues can help fire prevention stakeholders better understand the feasibility and desirability of their practical application to wildfire resilience.

\section{California}

Conventional means of wildfire mitigation associated with the transmission system have been found lacking. For example, in California, following years of major wildfires that included interactions with the electric system, distribution companies are deploying preventive de-energization of the grid during hot, dry high-wind conditions to prevent the power system from igniting wildfires. Though unpopular by those losing service, these outages, known as public safety power shutoffs (PSPSs), reflect that the highest priority of any power network entity is safe operation despite the significant costs.

Without adequate mitigating measures, such as local backup generation or microgrids, this can lead to customers being left without essential services, potentially for days. The hot, dry weather is common in the late summer and fall. The high, turbulent, and dry wind tends to trigger PSPS events, which typically last one to two days but can stretch to several days in hard-to-restore areas. PSPSs act as an emergency measure in place of longer-term investments such as reconductoring, reinforcement, undergrounding, or introduction of new technologies such as distributed storage.

The reason such disruptive actions become necessary is the extreme cost and devastation wildfires can cause when started by grid failures. One notable example was the Camp Fire on Nov. 8, 2018, in the northeast of the state. This wildfire, caused by the failure of a hook carrying a conductor, led to an estimated \$17 billion (USD) in damages, including 85 fatalities, 18,804 lost structures, $620 \mathrm{~km}^{2}$ of burned area, and billions of dollars in fines and liabilities for Pacific Gas \& Electric Company (PG\&E). 
The company fell into bankruptcy following a history of similar, damaging events. Its stock price fell by $66 \%$ over 13 months, although it was able to exit bankruptcy in June 2020 after agreeing to a range of settlements with government agencies, insurance companies, and other claimants. A victims' fund was established with $\$ 5.4$ billion in cash and $22 \%$ of PG\&E's stock. PG\&E is undertaking a safety program that is expected to cost about $\$ 6$ billion during 2021 and 2022 and has proposed a long-run goal of undergrounding about a tenth of its system, about $16,000 \mathrm{~km}$ of lines, for $\$ 15$ billion to $\$ 20$ billion. Even critics contest this estimate as too low since much of this work would be in remote, unpopulated, and rocky areas.

In 2021, PG\&E also began various other programs to mitigate the impact of outages, such as distributing batteries or subsidized generators to vulnerable customers. On the other hand, widespread use of local generators can be a hazard and damage air quality. Also, if fuel is poorly or unsafely stored in high fire risk areas, it could act as an accelerant. The transport of replacement fuel during wildfires can also be hazardous and unreliable. Consequently, sites wanting high resilience often invest in large underground storage.

Given California's history of wildfires associated with power grids and increasing propensity to use PSPSs, independent microgrid technologies being used by both communities and individual customers in the "wine country" appear increasingly attractive.

Blue Lake Rancheria (BLR) has a demonstration microgrid implemented by the Blue Lake Tribe together with Humboldt State University (Figure 1). The microgrid includes $420 \mathrm{~kW}$ of PV, a 500 $\mathrm{kW} / 950 \mathrm{kWh}$ battery bank, and a $1 \mathrm{MW}$ backup generator, all connected to the PG\&E distribution grid at $12.5 \mathrm{kV}$ through a computer-controlled circuit breaker. A fueling station and attached convenience store form a second microgrid with PV, batteries, and some building system controls. The main microgrid system was not initially designed to be a preventive measure against wildfires, but was motivated by the tribe's sustainability culture and potential cost savings of about $\$ 200,000$ (USD) a year. 


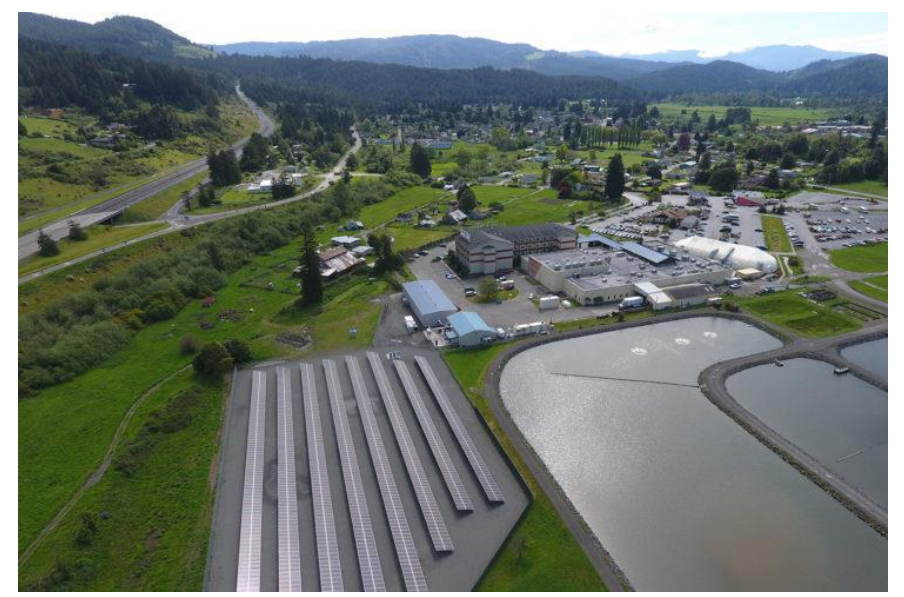

Figure 1: Overview of Blue Lake Rancheria casino complex.

(source: Schatz Energy Research Center, Humboldt State University).

As PSPSs are increasingly necessary, customers connected to a microgrid retain access to services and supplies, such as fuel, ice, internet connection, electronic-device charging, and ATMs. Also, the Blue Lake Tribe's event center and hotel can house vulnerable evacuees in an emergency. The microgrid acts as a hedge against failures of the bulk transmission system and supports the wider resilience efforts to control wildfire risk as part of a portfolio of wider actions, which may be taken by planners and operators. The hotel microgrid was first activated for a fire threat in October 2017 (Figure 2). The microgrid islanded when a small brush fire started nearby, and the hotel acted as a shelter for evacuees and a command center for emergency crews.

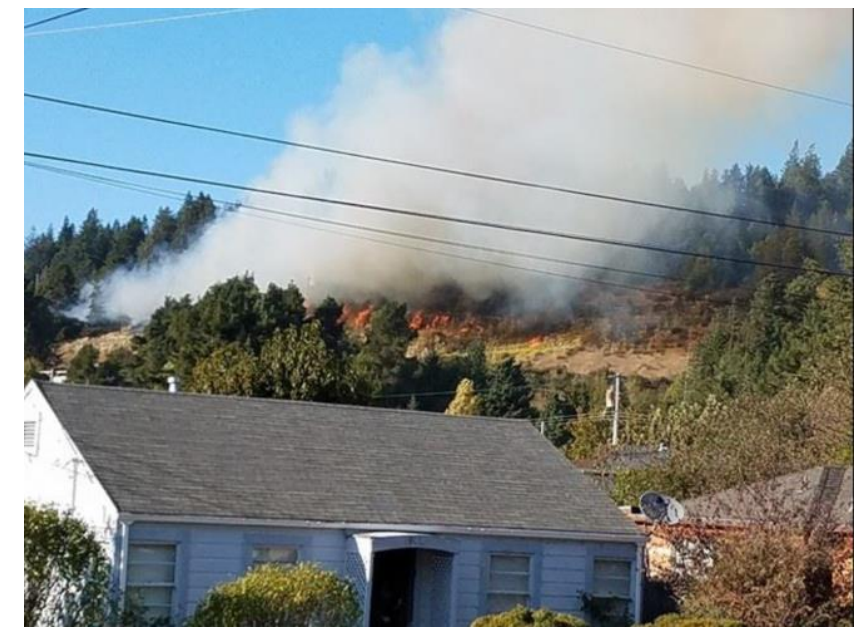

Figure 2: Fire Near Blue Lake Rancheria.

(source: Redheaded Blackbelt, 8 October 2017).

Regulatory Response

Microgrid deployment has a recurring pattern. They are installed for a variety of reasons, but after they exhibit excellent resilience performance during an emergency, they become promoted primarily 
for resilience. Following Superstorm Sandy in 2012, microgrids became heavily promoted for community safety in the northeastern United States. Several states have microgrid deployment programs, such as the New York Prize. California similarly had a notable microgrid research program in place, including BLR described above, but relatively slow deployment beyond the demonstration phase. Fires, though, have proven to be this state's microgrid motivator. A few examples of notable resilience performance, including $B L R$, pushed microgrid development to the fore to mitigate the consequences.

In September 2018, the California Legislature passed SB 1339. This bill requires the California Public Utilities Commission (CPUC) to address some of the barriers impeding the deployment of microgrids. Californians had recognized the severity of the deteriorating fire situation, so the CPUC made its first order of business establishing exemplar microgrids such as BLR that could contribute to resiliency before the summer of 2020. Companies were ordered to:

- develop and implement standardized pre-approved system designs for interconnection of resiliency projects that deliver emergency services

- develop and implement methods to increase simplicity and transparency of project approval; and

- prioritize interconnection of resiliency projects for key stakeholders

Further, several temporary mobile substation generator projects were identified, and PG\&E was encouraged to develop them as soon as possible. A community microgrid program was also established.

During the second phase, in January 2021, the CPUC addressed regulatory barriers to microgrid deployment. Notable was the requirement that a limited number of microgrids be permitted to serve neighboring sites' critical loads while not being subject to utility regulation. This move weakens a historically formidable barrier to microgrid development, namely that the microgrid might then be considered a public utility and be subject to overly burdensome regulation. Utilities were also directed to develop standard microgrid tariffs, pilot demonstration projects, and an incentive program.

Most recently, in July 2021, the CPUC suspended a key provision of tariffs to allow a microgrid to be charged for the required utility provision of backup to the microgrid's generation under some circumstances. The CPUC intends to wade into the controversial topic of main grid provision of fallback for microgrids. 


\section{Other examples around the globe}

\section{Greece}

Other noticeable examples of the deployment of DER to support reactive recovery from wildfires include that from Greece, where severe forest fires between Aug. 24 and Sept. 7, 2007, caused considerable damage to the power system with 2,500 burnt poles and disrupted supply to 90,000 customers. The restoration of over $20 \%$ of affected customers' electricity supply took more than five days.

In response to this, distributed energy systems, including mobile diesel generators of between 50 and 130-kVa, were used for restoring power supply to parts of the distribution network, forming ad-hoc low-voltage microgrids. Had microgrids been more widely deployed or more extensive sets of resources been available, such extreme recovery times may have been significantly ameliorated. The addition of DERs, such as solar photovoltaic or micro-wind, could also serve to expand the capacity of microgrids, particularly in regions with large solar resources such as Greece and southern Europe more generally.

Following an extreme heatwave with the highest temperatures reaching $47.1^{\circ} \mathrm{C}\left(116.8^{\circ} \mathrm{F}\right)$ in August 2021, a series of wildfires erupted, where 125,000 hectares of forest and cultivable land and dozens of homes burned for more than three weeks. The number of fires was $26 \%$ above the average of the past 12 years, and the area burned was bigger on average by $450 \%$. The largest wildfires were in Attica, Olympia, Messinia, and the most destructive in northern Evia, with over 50,000 hectares burnt, which is nearly a quarter of the island. Figure 3 shows the wildfires in Greece according to the NASA wildfire tracker from Aug. 2-8, 2021. Figure 4 shows an example of some of the mobile generators being deployed. 


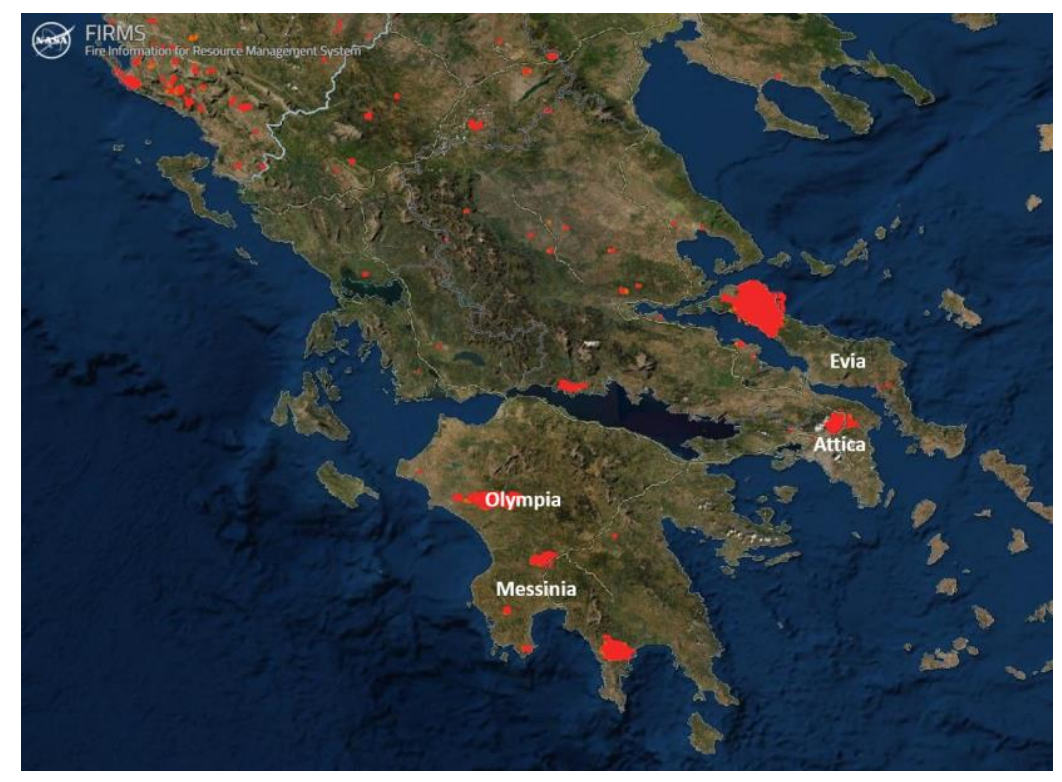

Figure 3: Wildfires in Greece from Aug. 2- 8, 2021 (source: NASA wildfire tracker).

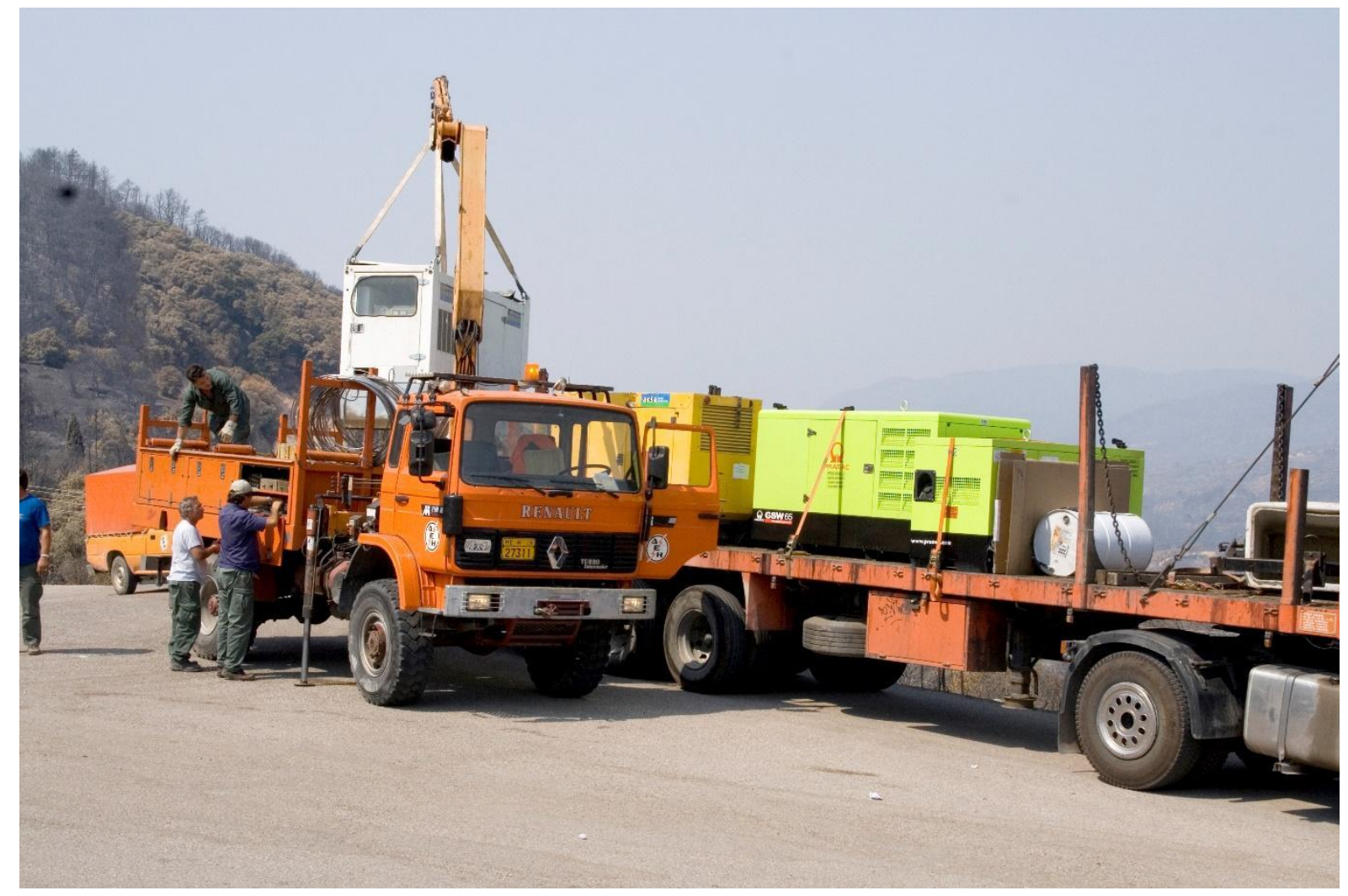

Figure 4: Use of mobile generators for restoration (Courtesy: Public Power Corporation, Greece).

In all areas, network damages were extensive. In Evia, $7 \mathrm{MV}$ lines feeding electricity to 13.000 consumers were destroyed. In Attica, $9 \mathrm{MV}$ lines were affected that electrify 38,000 consumers. 
Twelve mobile generators with a total capacity of $2.12 \mathrm{MW}$ were used to provide the essential electricity needs before network restoration.

Despite the severity and geographical dispersion of these damages, the Greek DSO (HEDNO) managed to restore electricity to $98 \%$ to $100 \%$ of the affected clients within 10 days. The only exceptions concerned individual houses and small remote settlements. This fast recovery would not have been achieved by restoring the network alone. Mobile diesel engines were extensively used at secondary substations to supply parts of undamaged LV networks and supply individual installations. Diesel units were also installed in all critical water pumping stations and in dispersed locations to supply parts of the healthy networks.

\section{Australia}

In Australia, the Resilient Energy Collective is using prebuilt equipment to restore supply in communities on a micro-scale following bushfires. This is a more responsive and reactive approach to mitigate the impacts of wildfires, but it could be vital to restoring life-critical loads, especially in hot regions which can suffer extreme heat, and exacerbating issues such as food spoilage and water loss due to non-functional water pumps. Australia, at the same time, has the benefit of significant solar resources. Although dense smoke can limit photovoltaic (PV) output, this places Australia in a good position to utilize distributed solar energy resources, especially in areas that may have more sparse access to the grid or be particularly vulnerable to bushfires.

The Victoria towns of Donald and Tarnagulla also provide remarkable examples of microgrid deployment. As these towns are at high risk of being cut off from grid supply in the case of bushfire (as well as other extreme weather events such as flooding), a feasibility study is considering the development of a DER-based microgrid that could exploit PV, battery storage, diesel generators, and intelligent control of various non-essential loads enabled by smart meters. To assess the resilience value potentially brought by the microgrid, as well as design the optimal local DER portfolio in conjunction with potential network augmentation, a risk-based probabilistic techno-economic framework is being adopted. Part of the challenge is to step out of specific sandbox setups and allow the adoption of this kind of advanced planning methodologies as regulatory business as usual.

\section{Others}

The deployment of microgrids is becoming an attractive solution against wildfires and extreme events around the globe. In Canada, there are various incentive programs (e.g., the Green Municipal Fund by 
the Federation of Canadian Municipalities) across the provinces to encourage communities to invest in sustainable, renewable-based microgrids. This includes both grid-connected and off-grid installations in indigenous communities which face serious challenges with secure electricity supply during extreme events.

Hawaii Electric Light Company promotes microgrid development to protect remote communities that might isolate from the grid in the face of severe events on the island, such as fires, volcano eruptions, and hurricanes.

Following the 2011 Great East Japan earthquake and tsunami, Japan accelerated its microgrid program. Microgrids were promoted for schools, industrial facilities, and emergency services, and a notable community system was installed at Higashi Matsushima.

These real-world examples and initiatives illustrate both the opportunities and challenges associated with using DERs and microgrids for resilience. The increasing realization that this is becoming the "new norm" as a direct impact of climate change, with longer heat and drought periods, has pushed key stakeholders and decision-making bodies to take the issue more seriously than ever before.

The following sections provide a systematic discussion on the role and beneficial utilization of DERs exploited through microgrids under the wildfire threat and how DER investments can be planned to effectively complement network investments against wildfires.

\section{Utilization of DERs under wildfire threat}

In case of a progressing wildfire, the DERs of an endangered power system and mobile power sources can be utilized to minimize load shedding due to wildfire impact on system components. For instance, the schedule of flexible loads and placement of mobile energy resources can be determined considering the system components affected by the wildfire and when they are expected to be affected. This section presents actions that can be taken by a system operator to mitigate the impact of a progressing wildfire on the electrical system and minimize customer interruptions.

To detect a wildfire and estimate its scale and progression, a situational awareness system is necessary. The information from such a system can help assess the spatiotemporal impact on each system component. An effective assessment of the affected components is useful not only for determining the emergency operational response, but also for the restoration phase as it allows the system operator to develop an efficient restoration strategy. 
Before the event, camera networks, combined with machine learning techniques for image analysis, can automatically detect a wildfire outbreak. Once a wildfire is detected or reported, its propagation can be assessed. Using the spatial data of an endangered system, e.g., by a geographic information system (GIS), the distance between the wildfire and the conductors or transformers can be estimated during the wildfire's progression. Based on that estimate, the wildfire's impact on them can be assessed. For instance, the temperature of any line can be estimated. Therefore, its status can be evaluated to determine the operation of system assets to minimize the approaching wildfire's impact. If part of the system is expected to be isolated due to line-related damage, the operator prepares for its smooth operation and the uninterrupted supply for consumers by forming self-sufficient microgrids whenever possible.

Wildfires spread depending on various factors, including weather parameters (e.g., wind speed and direction and relative humidity), the vegetation of the crossing area, terrain slope, fuel bed conditions, and flame front properties. Hence, weather forecasting tools and systems (e.g., satellite observations) for acquiring knowledge of territory characteristics are vital. Wildfire tracking is also important as collected data during wildfire progression can be used to update propagation assessment and organize system emergency responses. Satellites and unmanned aerial vehicles can be used to monitor wildfire progression when approaching the fire is unsafe.

There are a variety of tools available to estimate wildfire propagation. PG\&E uses a tool developed by Technosylva to derive fire propagation and consequence outcomes, such as impacted infrastructure. Additionally, the U.S. Forest Service uses the FlamMap application which includes the FARSITE simulator to compute wildfire growth and behavior. The tool considers detailed sequences of weather conditions. Since propagation depends on weather forecasting and terrain characteristics that cannot be quantified with great accuracy, different scenarios can be explored by considering the forecast error and quantification accuracy of terrain characteristics. PG\&E highlighted the need to use a probabilistic fire spread model.

Once the timing and impact of wildfire on system components is assessed, a preemptive operation strategy can be determined. Customer interruptions may be mitigated through the schedule and dispatch of the DERs and network reconfiguration, taking into account the stochastic generation of renewables. Wildfires mainly affect overhead lines, poles, and substations components. Concerning overhead lines, a wildfire can damage them or erode their thermal rating due to the increase of the conductor's surface temperature. Also, the impact of wildfire on DERs can be estimated to develop a strategy based on the available generation units. DERs can be fully destroyed by the wildfire or their 
operation can be affected by its smoke. For instance, wildfire smoke contains small particles that reduce the amount of sunlight reaching solar panels, reducing the PV output.

To mitigate issues expected from a reduction in a conductor's safe carrying capacity, the system can be reconfigured with DERs and other resources operated to avoid limit violation and tripping. If line damage is expected, the system operator needs to determine a system reconfiguration to maintain the full connectivity of the system. If full connectivity is not possible, appropriate schedule and dispatch of the DERs may minimize load shedding in the isolated parts of the system and ensure uninterruptible system operation. If the wildfire damages a substation, load shedding of downstream loads can be mitigated by using the residential DERs (including flexible loads) connected to the low voltage distribution system.

Figure 5 shows damage provoked by the 2007 forest fires in Greece. In the case of Greece wildfires, monitoring the spatiotemporal impact of the wildfires via GIS has assisted the distribution system operator in choosing appropriate locations for the mobile generators to reduce restoration times after the damage. GIS information also enables the rapid development of restoration plans. The timely estimation of the required number of repair crews and necessary materials for system restoration significantly shortens system restoration times. A tool for estimating wildfire propagation was not available in Greece. Such a tool could support faster decisions to identify which buses to connect the mobile generators, further reducing customer interruptions.
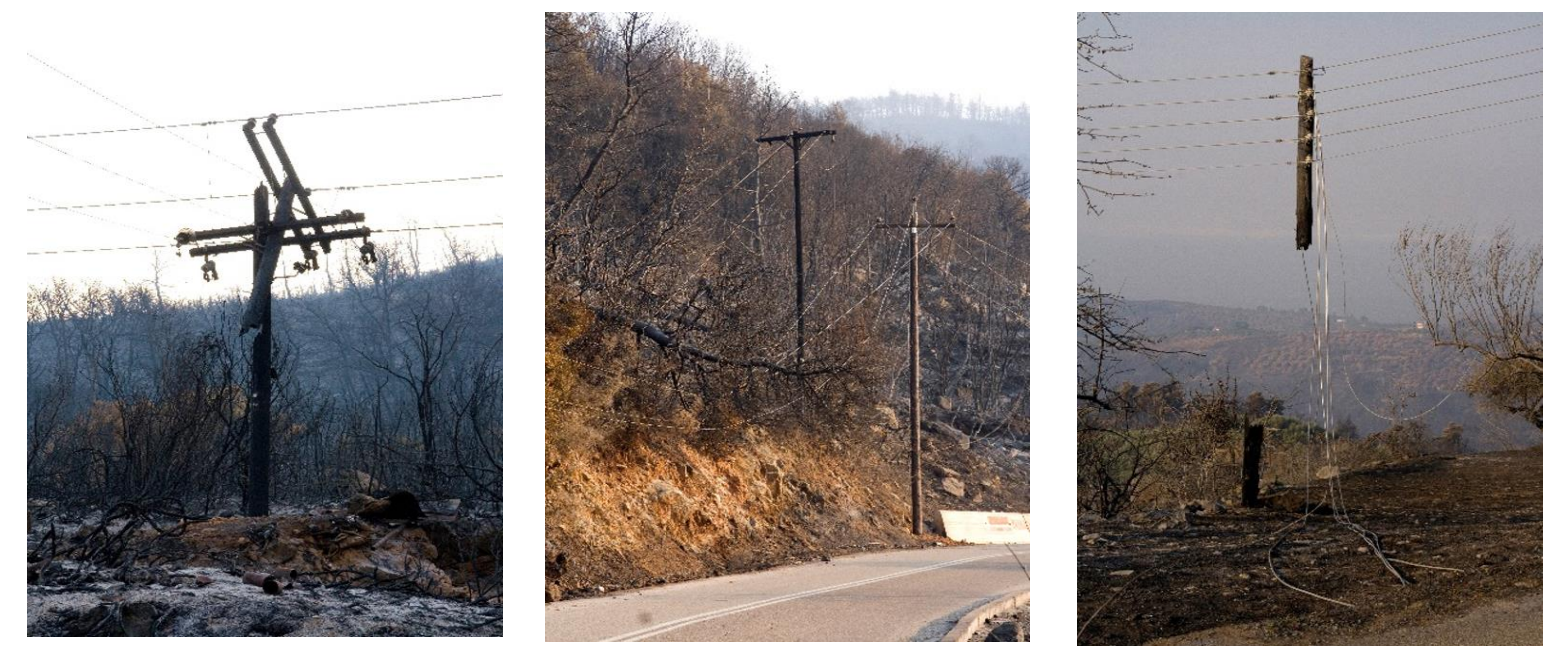

Figure 5: Damaged lines and poles from the 2007 forest fires in Greece (Courtesy: Public Power Corporation, Greece). 
If a situational awareness system is unavailable, and therefore, propagation of the wildfire and its impact on system components cannot be assessed, DERs can be scheduled and dispatched to form smaller self-adequate microgrids. In this way, when a line is set offline due to the wildfire, the downstream system can survive with minimum load shedding as it is already organized as a selfadequate microgrid able to meet the demand individually. However, the system reconfiguration, schedule of the flexible loads, state of charge of the energy storage system, and the placement of mobile energy resources cannot be determined optimally without the information provided by a situational awareness system.

In addition to preventive actions, proper corrective actions are necessary to mitigate the impact on the system during the restoration phase. Once the wildfire has been extinguished and the damaged components have been identified, the development of a restoration plan, including the damage repair sequence, network reconfiguration, and utilization of DERs, is highly important. The order of repairs can be based on the capability of DERs to meet demand in the isolated areas of the system. For instance, if the DERs within an isolated area can meet the demand for a long period, the damages that led to the isolation of this area can be repaired last. Also, the routing of mobile resources can be coordinated with the repair sequence. Mobile resources can be used to mitigate the load shedding of the isolated areas until the damaged components are restored. In this case, the status of the transportation network is also taken into account to determine the routing of mobile resources. 


\section{Planning DER investments against wildfires}

Most parts of the electrical network were built decades ago, and, in many cases, without considering natural threats such as wildfires. Today, several critical network infrastructures and supply/entry points to distribution networks are highly exposed to natural hazards. The severe impact of wildfires on the electricity supply demonstrates the need to prepare against such future events.

A well-established body of recent work has been dedicated to calculating the probability of large wildfires. The Keetch-Byram Drought Index, the Fire Potential Index, and the Large Fire Probability, which have been widely used in the United States, are good examples of metrics to identify areas with wildfire potential. In another example, shown in Figure 6, the Chilean forest authority uses the Forest Fire Ignition Probability Map for the same purpose. This probability is computed daily based on solar radiation, temperature, and dead fine-fuel moisture.

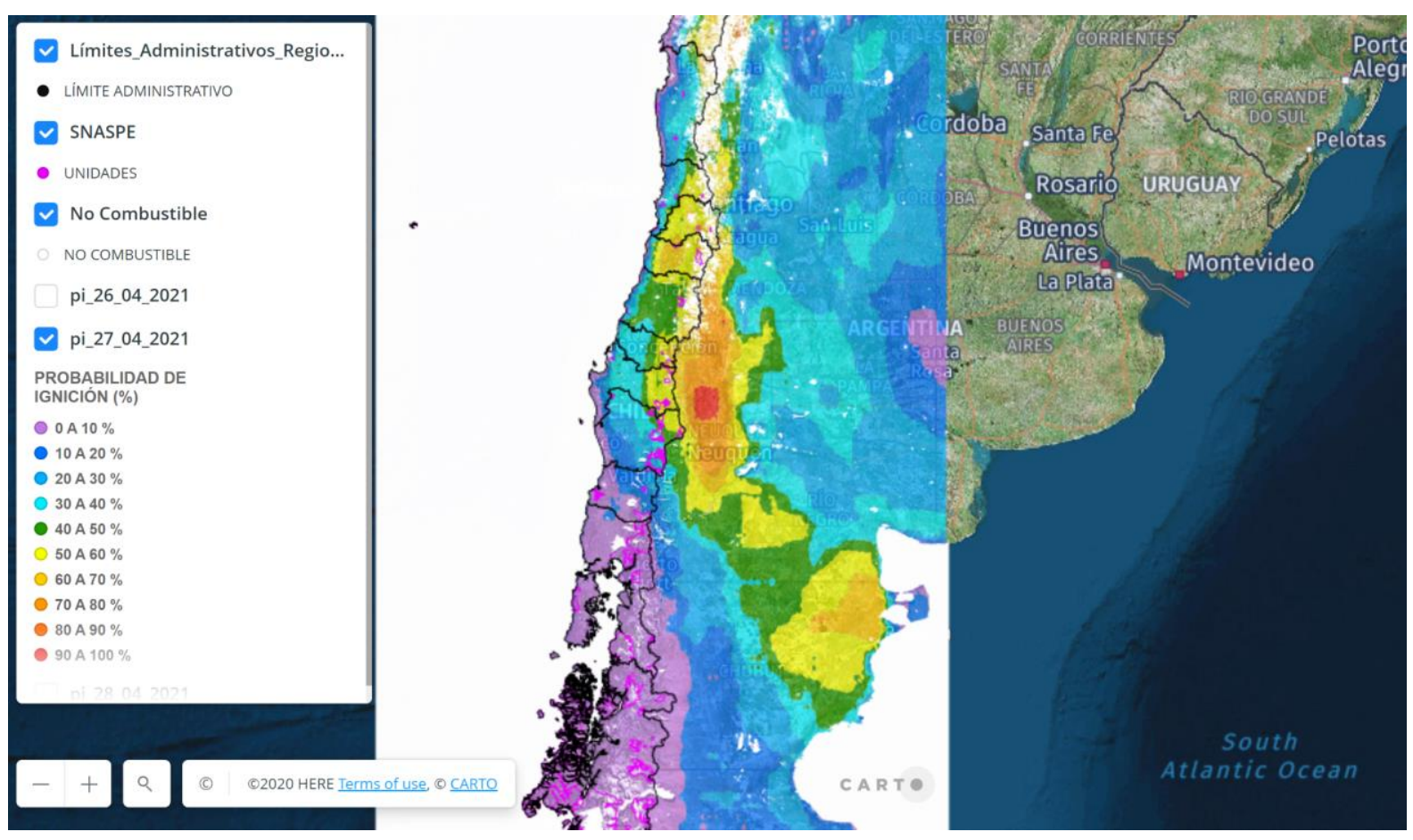

Figure 6: Forest Fire Ignition Probability Map (April 27, 2021).

Source: https://geprif.carto.com/

Apart from these probabilities, historical data is available regarding the duration of large wildfire events and repair times of the faulted electrical infrastructure after such catastrophic events. Also, from recent experiences in Chile, the power network may be impacted in several points simultaneously by a large wildfire event (Figure 7). Under these conditions with lost supply points from the main grid, an optimal DER portfolio could be used to supply the internal loads. 


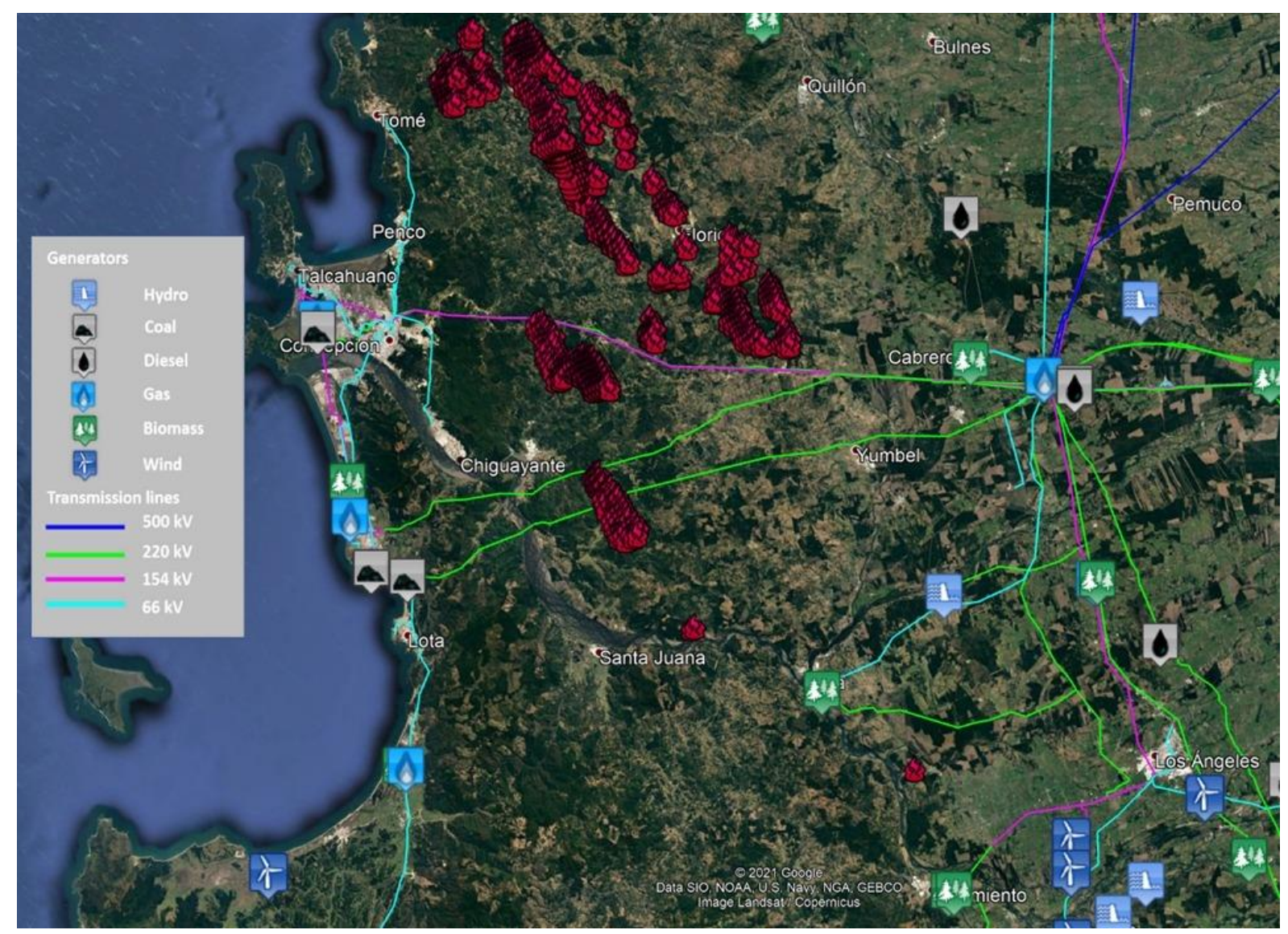

Figure 7: Representation of wildfires in Chile on Jan. 26, 2017.

Planning an optimal DER portfolio against large wildfires requires a suitable methodology that considers several relevant uncertainties. The following presents a proposed methodology for resilient network design, used in the "Review of Distribution Network Security Standards" and now under development in the United Kingdom. A similar risk-based probabilistic techno-economic framework is being used to inform policymakers and industry in Chile and Australia.

\section{A suitable methodology to design DER portfolios against wildfires}

The following methodology seeks optimal investments in DER portfolios to efficiently hedge a distribution system against high-impact low-probability events such as wildfires. The methodology is based on an optimization model and, importantly, also allows capturing the substitution effect between DER and network investments since it co-optimizes both. In operational timescales, the model determines the optimal operation of the distribution system with DERs, including purchases from the main grid and topology control. Hence, the optimization model can identify optimal 
preventive and corrective measures to hedge the distribution system against potential outage scenarios originated from wildfires. The model optimizes the following set of decisions:

- Preventive measures: Investments in DER equipment such as storage plants, backup generation, and network investments. The model also finds the optimal volume of demand response contracted. These measures are made upfront, pre-contingency, and thus are present in all scenarios.

- Corrective measures: These measures depend on the specific contingency and are scenario dependent. We model two types of corrective measures, fast and slow:

- Fast: Refers to the distribution system operation itself, including demand curtailments and a (smart) operation of system assets (topology control and dispatchable DER). These actions can occur right after a contingency occurs.

- Slow: Installing and dispatching mobile DER. These actions feature a lag associated with the arrival of mobile equipment.

The proposed optimization model is probabilistic, minimizing expected costs (including the cost of investment, operation, and energy not supplied, and, eventually, a risk metric to capture risk aversion). It also considers the occurrence of several scenarios (each with a probability) in the form of a comprehensive set of system outages, including those triggered by wildfires. Importantly, in the event of a wildfire, the probability of simultaneous outages becomes high since a single fire event can affect various pieces of system equipment. Here, ignition probability maps as those in Figure 6 (and other risk indices associated with wildfires discussed earlier) can inform about the places with the highest risks of wildfires.

\section{Illustrative case study example}

The model above was applied to the textbook-like system design example displayed in Figure 8. This example was used in the "Review of Distribution Network Security Standards" in the United Kingdom to illustrate, from a fundamental viewpoint, the problems of the current network standards and the potential solutions going forward. This example seeks to determine, in a greenfield fashion, the optimal system design to supply areas A and B. The figure shows all candidate assets (i.e., investment propositions) to supply the constant loads in the two distribution networks (25 MW in Area A and 50 MW in Area B). The set of candidate assets includes six power lines and distributed PV and battery systems in Area A. In case of network outages, the choices of renting mobile generation units and exercising DR contracts in Area A and B as corrective actions could also be considered as part of the system design. Importantly, the failure rates of lines 5 and 6 are affected by the risk of wildfires, which 
increases the probabilities of failures in that network corridor and originates dependencies of line failure probabilities between lines 5 and 6 .

Under normal conditions, the outage rate of lines is 1 occurrence per year (occ/yr) with a mean time to repair of one day. Under catastrophic wildfire conditions in the neighboring area of lines 5 and 6 (1 occurrence in 10 years), we assume these lines will fail (simultaneously) and that their mean time to repair increases to 30 days. Other relevant input data include an energy price of $\$ 50 / \mathrm{MWh}$ (use to buy energy from the main grid), a value of lost load (VoLL) equal to $\$ 10,000 / \mathrm{MWh}$, and the investment costs of lines, PV, and storage of $\$ 75 / \mathrm{MW} . \mathrm{km} . \mathrm{yr}, \$ 500 / \mathrm{kW}$, and $\$ 200 / \mathrm{kWh}$, respectively.

Regarding the mobile generation units in both areas, the system operator can rent them at an hourly cost of $\$ 68.5 / \mathrm{MW}$ and operate them with a $\$ 200 / \mathrm{MWh}$ fuel cost. For simplicity, DR features the same costs as those of mobile units. We also assume that the system operator takes an average of 2.4 hours and three days, respectively, to install the mobile units under normal and wildfire conditions. Mobile units and DR measures can cope with up to half the power demand in each area.

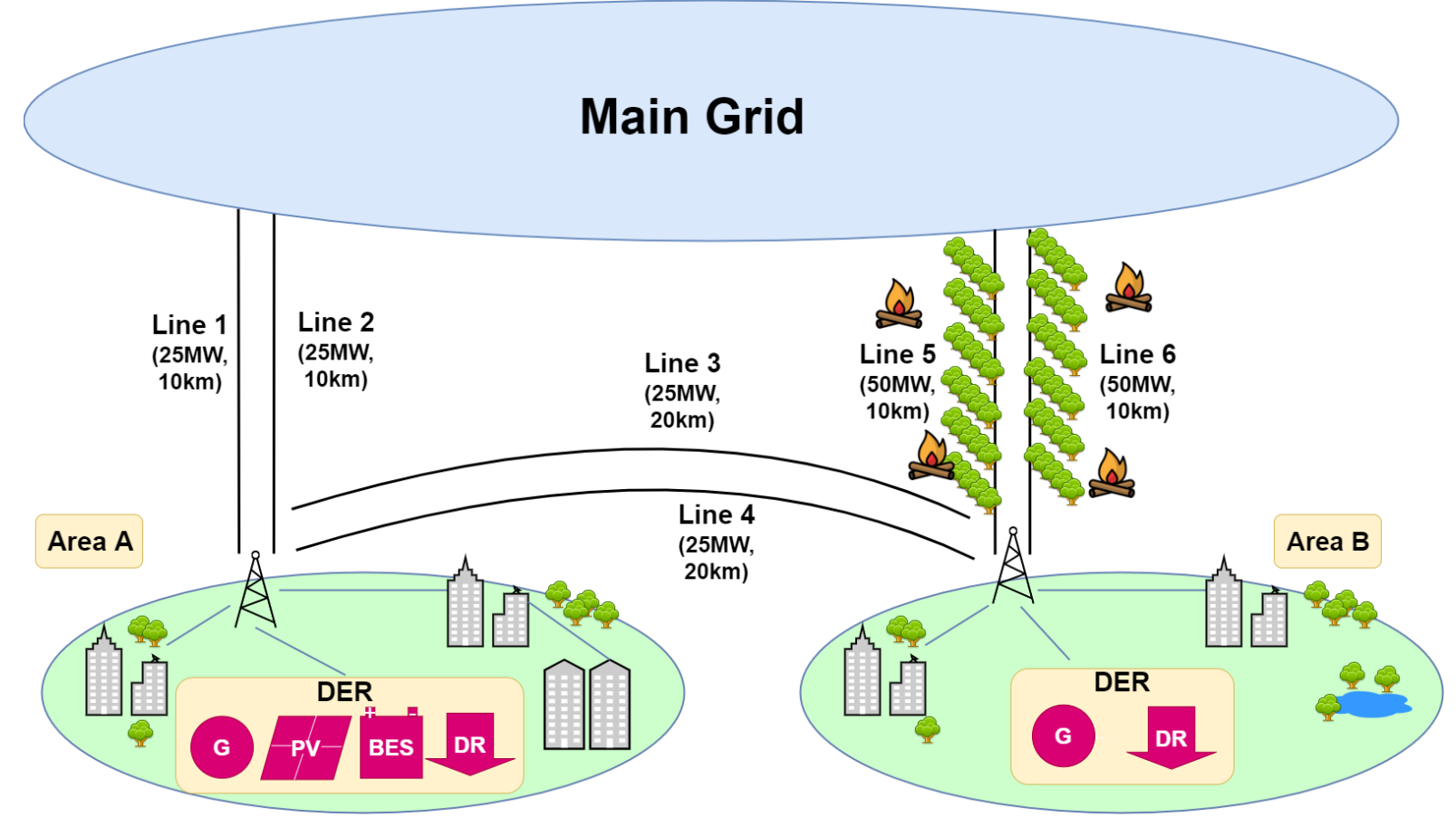

Figure 8\}: Electricity network and DER candidates along with areas exposed to wildfires.

We consider 22 scenarios, including one intact system (with no outages), six N-1 line outages, and 15 $\mathrm{N}-2$ line outages. The probabilities of these scenarios are calculated assuming independence and dependence under normal and wildfire conditions, respectively. Hence, once a wildfire occurs (every 
10 years on average), a common mode failure arises, affecting lines 5 and 6 simultaneously. The probabilities with and without the risk of wildfires are shown in Table 1. Notably, the probability of a double contingency affecting lines 5 and 6 increases by more than 110,000\% when we assume a wildfire every 10 years (on average).

Table 1: Scenario probabilities without and with the risk of wildfires. Dependent probabilities have been marginalized (using the fraction of the time to which the network is exposed to a wildfire).

\begin{tabular}{l|rrr}
\multicolumn{1}{c}{} & \multicolumn{2}{c}{ Probabilities } & \\
\cline { 2 - 4 } & Independent & Dependent & \multicolumn{1}{c}{ Variation } \\
\cline { 2 - 4 } Intact system & $9.84 \mathrm{E}-01$ & $9.76 \mathrm{E}-01$ & $-1 \%$ \\
All N-1 failures & $1.62 \mathrm{E}-02$ & $1.60 \mathrm{E}-02$ & $-1 \%$ \\
All N-2 failures but L5-6 & $1.03 \mathrm{E}-04$ & $1.03 \mathrm{E}-04$ & $-1 \%$ \\
Double failure of L5-6 & $7.38 \mathrm{E}-06$ & $8.16 \mathrm{E}-03$ & $110404 \%$ \\
All failures beyond N-2 & $4.05 \mathrm{E}-07$ & $4.02 \mathrm{E}-07$ & $-1 \%$
\end{tabular}

Note that knowing the actual probability distribution functions of rare events such as wildfires may be difficult. Nevertheless, the probabilistic framework may still be beneficial for deriving good estimates when certain probability values are assumed. Alternatively, other optimization frameworks (not referenced here) that do not rely on probability values may be promising for resilience analysis.

Table 2 shows the results found for three cases, namely, Case A, Case A Re-evaluated, and Case B. Case A corresponds to the results when the risk of wildfires in the neighboring area of lines 5 and 6 is neglected (i.e., independent probabilities used in the optimization). Case A Re-evaluated features the same infrastructure as Case A, where the costs of operation and unserved energy have been reevaluated, including the risk of wildfires (i.e., independent probabilities used in the optimization and dependent probabilities used in the re-evaluation). Case B corresponds to the optimal design when the risk of wildfires is appropriately considered (i.e., dependent rather than independent probabilities have been considered in the optimization). 
Table 2: Results with costs in thousand dollars (k\$) per year. L, MG, DR, PV, and BES refer to line, mobile generator, demand response, PV panels, and battery energy storage, respectively.

\begin{tabular}{|c|c|c|c|}
\hline & Case A & $\begin{array}{c}\text { Case A } \\
\text { (Re-evaluated) }\end{array}$ & Case B \\
\hline Assets and measures & $\begin{array}{c}\mathrm{L} 1, \mathrm{~L} 2, \mathrm{~L} 5, \mathrm{~L} 6, \mathrm{MG}, \\
\mathrm{DR}\end{array}$ & $\begin{array}{c}\mathrm{L} 1, \mathrm{~L} 2, \mathrm{~L} 5, \mathrm{~L} 6, \mathrm{MG}, \\
\mathrm{DR}\end{array}$ & $\begin{array}{l}\text { L1, L2, L3, L4, L5, } \\
\text { PV, BES, MG, DR }\end{array}$ \\
\hline PV+BES investment cost & - & - & 11,500 \\
\hline Line investment cost & 113 & 113 & 150 \\
\hline Operational cost & 32,850 & 33,115 & 21,901 \\
\hline Lost load cost & 27 & 19,665 & 6 \\
\hline Total cost & 32,990 & 52,893 & 33,558 \\
\hline
\end{tabular}

\section{The Classic N-1 design}

Suppose we neglect the risk of wildfires in the neighboring area of lines 5 and 6 . In that case, the optimal system design found by the probabilistic model will be the classic $\mathrm{N}-1$ configuration, installing lines 1 and 2 (25 MW each) and lines 5 and 6 (50 MW each) without installing up-front DER capacity (Case A in Table 2). Interestingly, mobile generators and DR in both areas can be used as corrective actions to mitigate the impacts of $\mathrm{N}-2$ line outages and thus supply part of the load.

\section{The resilient design}

Suppose we consider the risk of wildfires in the area in question. In that case, the optimal system design will include a richer set of DERs, combining up-front investments in PVs and battery systems with corrective actions in the form of mobile generating units and DR (Case B in Table 2). Remarkably, the consideration of this richer set of DERs will reduce line investments through the risky network corridor (investment in line 6 is dropped) and increase investments in lines to transfer power between areas $A$ and $B$ (lines 3 and 4). This resilient design solution will limit the lost-load cost to only $\$ 6,000$ per year (which is significantly smaller than the lost-load cost of the other solutions), including the unserved demand during wildfires. Another remarkable result is that the PVs and battery systems, triggered by increasing the risk of wildfire from no occurrence to 1 occurrence in 10 years, are also used to reduce the power imported from the main grid under normal conditions, decreasing operational costs. These results demonstrate the multiple benefits of DERs and the importance of capturing these in the cost-benefit analysis to justify investments. 


\section{The actual risks associated with the current security standards}

Table 2 conveys another lesson. The actual risk to which the system is exposed due to applying the current security standards is significant. Indeed, the expected unsupplied energy cost of Case A REevaluated is 700 times higher than the initial evaluation (see differences between Case $A$ and Case $A$ Re-evaluated), increasing the total cost of the system design to almost twice the first estimate. Instead, suppose wildfire risks were appropriately recognized from the beginning to plan the system accordingly (as in Case B). In that case, the increases in total costs of the resilient design will be almost negligible compared with the initial cost estimations of the classical N-1 design (see differences in total costs between Case A and Case B). This result demonstrates the importance of appropriate resilient planning.

\section{The way forward}

\section{Smart grid resilience}

The conventional electricity network is getting smarter, meaning that:

1. Generation, storage systems, and other energy resources will become increasingly distributed, as load control inherently is located closer to or at the demand points, (e.g., rooftop PV arrays).

2. Networks will employ more modern technology and become more active through new flexible systems (e.g., flexible alternating current transmission systems, HVDC, and other power electronic-based equipment, such as grid forming inverters). Also new monitoring, control, protection, information, and communication technologies will be deployed.

3. Demand will become more controllable, with consumers participating actively in market and system operations.

In this vein, transport and heating/cooling electrification will present prospects to capitalize on flexible and controllable loads, exploiting their virtual storage capabilities. In the future smart grid world, the digitalization of energy systems will provide unique opportunities for much smarter management of the electricity system during extreme events. For instance, this smart management can include switching off non-essential demand when the network is stressed while supplying essential demand. The supply of essential loads during emergencies will also be enabled by DERs and virtual storage capabilities from demand (e.g., battery units from electric vehicles). This would significantly enhance the resilience of supply delivered as energy consumers will have their essential load supplied during high-impact events, including wildfires and other natural catastrophes. 


\section{New regulatory arrangements for a resilience future}

Private, competitive agents could efficiently undertake investments in distributed generation and other DERs in a decentralized fashion if the market appropriately remunerated investors. Hence, a distributed energy resource enhancing resilience could be remunerated, for instance, in terms of the (marginal) benefits originated by the lost-load cost savings caused by its presence. Fostering resilience through pure market signals, though, may encounter practical problems as follows:

- The distribution network investment regime may not be compatible with the efficient deployment of DERs. Indeed, DERs may even compete against new network infrastructure (usually built in a regulated, mandated fashion) for services such as reliability and resilience. Hence, some form of coordination may be needed, promoting the right share of investments between wires and non-wires (DERs) solutions

- Prices in distribution networks do not reflect the actual locational marginal cost of energy, including those during scarcity conditions. In fact, for appropriate market-driven investments in DERs, prices in distribution networks during scarcity conditions (right after a threat occurs) should be equal to the VoLL precisely in those areas/feeders where demands are being curtailed. Given the extreme social conditions associated with natural hazards such as wildfires, it may be politically impractical to maintain prices equal to the VoLL under such circumstances

- Even if efficient pricing were in place for distribution networks, concerns remain regarding the performance of market-driven investments:

- Probability distribution functions of rare events such as wildfires are unknown and non-stationary due to climate change. Hence, a DER portfolio meant to hedge these risks would be difficult to justify on a market-driven basis.

- The above problem is exacerbated by the risk aversion of self-interest investors, who require more confidence about the revenue streams associated with their investments.

- Also, investors may act strategically to not fully provide a robust system design, preserving high prices in times of scarcity conditions.

These points suggest that pure markets will generally not deliver resilient DER solutions, and hence standards or mandates will be needed. However, part of the underlying problems associated with natural hazards, such as wildfires, remain in the centralized solution. A more centralized system planning problem will still need a definition of appropriate risk aversion levels and assumptions on partially unknown probabilities. This will require careful attention and, indeed, further research, 
mathematical models (particularly, optimization models under uncertainty), planning, and regulation going forward. In this vein, our previous examples and analyses can inform the development of future standards for resilience as in the United Kingdom, Chile, and Australia.

\section{Acknowledgment}

We acknowledge the support of Jorge Elizondo from Heila Technologies; Iraklis Menegatos, deputy chief executive officer of HEDNO, Greece; and the financial support of Agencia Nacional de Investigación y Desarrollo through grants Fondecyt/1181928 and Instituto Sistemas Complejos de Ingeniería PIA/APOYO AFB180003 and EPSRC, UK project IDLES (EP/R045518/1).

\section{Further reading}

- Zhou, Y., Panteli, M., Moreno, R., Mancarella, P., "System-level Assessment of Reliability and Resilience Provision from Microgrids", Applied Energy, Vol 230, pp 374-392, Nov. 2018.

- Moreno, R., Panteli, M., Mancarella, P., Rudnick, H., Lagos, T., Navarro, A., ... \& Araneda, J. C. (2020). From reliability to resilience: Planning the grid against the extremes. IEEE Power and Energy Magazine, 18(4), 41-53.

- Trakas, D.N., and Hatziargyriou, N.D. (2017). Optimal distribution system operation for enhancing resilience against wildfires. IEEE Transactions on Power Systems, 33(2), 2260-2271.

- Wang, Y., Rousis, A.O., and Strbac, G. (2020). On microgrids and resilience: A comprehensive review on modeling and operational strategies. Renewable and Sustainable Energy Reviews, $134,110313$.

- Strbac, G., Hatziargyriou, N., Lopes, J.P., Moreira, C., Dimeas, A., and Papadaskalopoulos, D. (2015). Microgrids: Enhancing the resilience of the European megagrid. IEEE Power and Energy Magazine, 13(3), 35-43.

\section{Biographies}

- Rodrigo Moreno is with the University of Chile, Instituto Sistemas Complejos de Ingeniería ISCI and Imperial College London.

- Dimitris N. Trakas is with the National Technical University of Athens.

- Magnus Jamieson is with the University of Strathclyde.

- Mathaios Panteli is with the University of Cyprus.

- Pierluigi Mancarella is with the University of Melbourne and the University of Manchester.

- Goran Strbac is with Imperial College London.

- Chris Marnay is with Lawrence Berkeley National Lab.

- Nikos Hatziargyriou is with the ex-chairman and CEO of Hellenic Distribution Network Operator (HEDNO). 